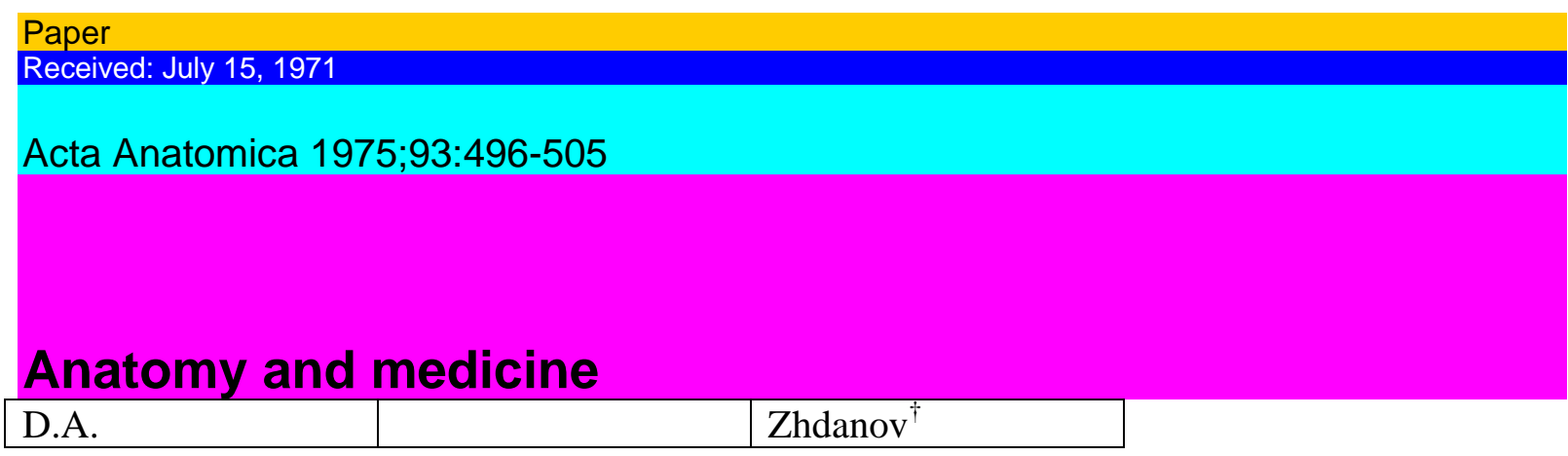

Department of Anatomy, 1st Moscow Medical Institute, Moscow, USSR

Anatomy has undergone tremendous changes in the 20th century due to the progress of the natural sciences and the development of new methods and tools of research based on the achievements of physics, chemistry and technology. It has become a science of living biological structures that are studied over a very wide range - from the molecular level to the level of the organism as a whole - in close relationship with the functioning and continuous alterations of these biological structures during evolution and individual development.

So present-day anatomy, as a science of the body structure as a whole, and of the structure of the component organs, tissues and cells as related to their functions in the course of genesis and development, is confronted with formidable and complicated tasks. It systematically describes the shapes and structures of organs, tissues and cells and subcellular elements with due consideration for their age and genital, individual and functional variability. It deals with the interrelationships of anatomical structures and their functions, the structure and position of the organs forming the organism, the physique as a whole, the tissues and cells during their genesis and development and in the course of evolution, primarily of vertebrate animals, and in human ontogeny. Such studies are aimed at bringing to light the extrinsic and intrinsic causes, influences and mechanisms which de-

1 Late President of the International Federation of Anatomists, Member of the USSR Academy of Medical Sciences and of the German Academy 'Leopoldina'. Head of the above Department. Received: July 15, 1971.

Zhdanov

497

termine the anatomical features of man as opposed to other animals, the mechanisms by which the organs and tissues adapt their structure to changing functional conditions, as well as ageassociated, sexual and individual variability.

The functional-anatomic, evolutionary-genetic and causal examination of the factual descriptive data and experimental evidence and the theoretical generalizations made by anatomy on this basis are of prime significance for the understanding of general biological laws and rules and for pathology and medicine in general. In 1796, Goethe put is quite aptly when he said: 'Die Anatomie gibt dem menschlichen Geiste Gelegenheit das Tote mit dem Lebenden, das Abgesonderte mit dem Zusammenhängenden, das Zerstörte mit dem Werdenden zu vergleichen, und eröffnet uns dieTiefender Natur mehr als jede andere Bemühung und Betrachtung'.

The extensive application of the experimental approach to the study of macro- and microstructures in different functional states has brought anatomy closer to physiology. The anatomy of today, armed with the electron microscope, autoradiography, and cyto-chemical techniques, is concerned with the morphological manifestations of the basic metabolic processes, 
which hitherto were amenable only to biochemical studies. At the submicroscopical level, anatomy now can see the visual images of molecular transformations of the living cellular material in its continuous dynamic development. That is how anatomy has come to be integrated with physiology and biochemistry in studying the biology of cells, tissues, organs and of the organism as a whole. For that reason, anatomy, together with physiology and biochemistry, constitutes one of the fundamentals for training the physician, who must understand the laws of human nature which are particular cases of the universal biological laws governing the lifes of organisms inhabiting the earth.

Indeed, Man, who stands at the top of the evolutionary ladder and whose body has been studied in great detail, acts like a mirror reflecting all the diverse aspects of nature. This has for long been well realized by the best minds of mankind. In 1794, Schiller wrote to Goethe who has then studying comparative anatomy: 'Von der ein-fachen Organisation steigen Sie, Schritt vor Schritt, zu den mehr verwickelten hinauf, um endlich die verwickeltste von alien, den Menschen, genetisch aus den Materialien des ganzen Naturgebäudes zu erbauen. Dadurch, dass Sie ihn der Natur gleichsam nacherschaf-

498

Zhdanov

fen, suchen Sie in seine verborgene Technik einzudringen' (Brief-wechsel, 23. August 1794).

We are engrossed by anatomy not only because it is part of the great science of life, biology, but also because it belongs to the fundamental of medicine.

It is difficult to overestimate the significance of anatomy for medicine. Since time immemorial it has been known that 'Cognitio corporis humani principium sermonis in arte medicinae'.

The importance of anatomy for medical theory and practice was well realized by the outstanding physicians of all times and peoples. At the beginning of the 19th century, the Russian physician and scholar, Yefrem Muichin, said that 'a doctor who is not an anatomist is not only useless but harmful'. And 100 years later, the distinguished Moscow clinician, Gubarev, also put it aptly when he remarked that 'without anatomy there is no surgery, no therapy, but only guessing and prejudices'.

Those who wish to reform medical education should remember that a precise knowledge of the human body form and structure is a condítío sine qua non for understanding the functions of the healthy or sick organism and, hence, for mastering the theory and practice of diagnosis and treatment. Similarly, knowledge of human anatomy is indispensible for designing appropriate preventive services, since a successful organization of preventive measures is impossible unless there is a clear understanding of the etiology and pathogenesis of the diseases. I would like to discuss this particular point in greater detail.

The great variations of form, structure and position of the organs as well as of the human physique as a whole are determined by numerous internal and environmental factors affecting the individual development. One must bear in mind not only the biological, but especially the social environment as well as the increasingly active influence of man upon his environment. Man alters nature in both a beneficial and detrimental manner. In so doing, he alters his own nature as well. It is one of the tasks of anatomy to investigate the intrinsic and extrinsic causes and factors determining the direction of human corporal development and the formation of individual features of the physique and of organ structure.

Such a causal approach to human body structure stems from the active attitude of medical science to the nature of man. Only by studying the structure of the human body and gaining a correct 


\section{Anatomy and medicine}

\section{9}

understanding of its development and of the ways in which hereditary information acts and the environment influences the organism, it is possible to perfect human body structure and functions, to make the organism stronger and healthier, to temper it in its fight against the harmful elements in the environment, and to prolong man's life. Hence, the direct ties between anatomy and preventive medicine. Personal and social hygiene rest on an understanding of the etiology and pathogenesis of diseases. Ivan Pavlov wrote that 'it is only through learning all the causes of diseases that the medicine of our days can become a medicine of the future, that is hygiene in the broadest sens of the word'. Looking into causes and mechanisms of morphogenetic processes, anatomy helps to understand the etiology and pathogenesis of diseases, because both normal and abnormal morphogeneses follow the same basic patterns, just as the physiology and biochemistry of the healthy and sick human organism are subject to the same basic rules. Incidentally, structural changes in organs under conditions of experimental pathology and in various human ailments in fact represent a kind of morphogenetic experiment, so that their study, like that of the effects on organ and tissue structure of various surgical and therapeutical interventions, furnishes the anatomist with most important information for testing on man the general morphogenetic rules, cell and tissue reactivity, and the adaptability of organ structure to variable conditions of functioning and existence. The similarity of the basic patterns followed by normal and pathological morphogenesis was clearly demonstrated in our studies in changes undergone by the lymph capillary bed under conditions of changed functioning or during experimental or natural abnormalities occurring in organs.

The latest research trends in anatomy and its achievements in the past few years have thrown open new possibilities for solving a number of problems of great significance not only for biology in general but also for pathology and medicine in particular.

Electron microscopy, especially when employed in conjunction with radioisotopes and other ultracytochemical methods, has extended the range of investigations into pathological processes from the cellular to the subcellular level, to the ever smaller cellular elements - up to millionth parts of a millimeter in size, that is, to macromolecules. This has given rise to molecular pathology which is 500

\section{Zhdanov}

evolving today as a new basis of medicine. The study of submicro-scopic manifestations of metabolism over a vast area of complex intracellular membrane surfaces provides important insights into the roles played by agranular reticulum enzymes in the lipoid metabolism, specifically in the intracellular esterification of cholesterol, in detoxication of poisons, and in the intracellular mechanism of drug actions.

Recent evolutionary studies at the cellular and subcellular levels carried out in the USSR using cytochemical and electron microscopic methods, have shown that the evolution of cellular structures and functions is subject to the phenomena of parallelism and divergence. Whether we are concerned with the evolution of the cell's heat resistance, the structural and functional organization of receptor cells of sense organs, or the characteristics of cytoplasmic proteins in different animals - more and more instances of parallelism and divergence are being discovered. The elementary mechanisms of tissue evolution have not yet been disclosed, and we do not know what intimate processes underly the unification of cells into a tissue and maintain tissue and 
organ integrity, nor what is the essence of the morphological, cytochemical and functional organization of tissues and organs under different conditions of existence and at different stages of phylogeny and ontogeny. The advent of new research tools should facilitate the resolution of these issues and thus raise evolutionary histology to a new height. This would have considerable implications for medicine and bionics.

The advances made by the qualitative and quantitative analysis of tissue, cellular and subcellular structures have made cyto- and histochemical methods more available for those concerned with the pathogenesis, diagnosis and even treatment of diseases. For example, cytochemical studies of II-hydroxylase in the adrenal cortex have established that the development of adrenogenital syndrome is associated with lack of the enzyme which is essential for converting chemical precursors to hydrocortisone, owing to which hormone synthesis proceeds along the path of androgens.

A remarkable achievement is the immunochemical determination of various hormones in the cells of the endocrine glands using the enzyme-labelled antibidy against a given hormone. In the thyroid, the enzymo-cytochemical assay for dehydrogenase glycerophosphate has revealed the so-called parafoUicular, or C-cells, which function

Anatomy and medicine

501

to elaborate thyrocalcitonin, a hormone reducing the calcium content in the blood.

Cytochemically, it has been found that cyclic adenosine mono-phosphate controls the activities of phosphorylase and glucose-6-phosphate dehydrogenase and thus provides the feedback from the trophic hormone-synthesizing cell to the targest cell from the respective endocrine gland. Our understanding of the pathogenesis of infectious and autoimmune diseases has been considerably advanced owing to the use of a broad range of immunocytochemical procedures (localization of viruses, bacterial antigens, tissue antigens, antibodies, tissue localization of sites of antigen-antibody interaction etc.).

A loss of correlation of nucleic acid and protein biosynthesis has been noted during malignant change in cells. An enzymatic characterization of metastases helps in determining the site of primary tumour nodes.

In the groups of hereditary diseases, certain histochemical procedures have come to be utilized as diagnostic tools. A fall in peroxidase activity in leukocytes has been found to be pathognomonic for an extra Y-chromosome. A rise in alkaline phosphatase in fibroblasts has been observed in trisomies and in Downe's syndrome. Conversely, a decreased activity of this enzyme has been shown to be associated with deletion of the 21st chromosome in humans with leukemia. Methods have been devised for the diagnosis of hereditary diseases through histochemical analysis of enzymes in microcultures from elements of the human hair bulb.

The application of histochemical methods for diagnostic purposes has been initiated in other fields of human pathology as well. For instance, the assay for phosphatase in hepatic cells is used in the differential analysis of obstructive jaundice and infectious hepatitis.

A histochemical analysis sometimes becomes the starting point for elaborating rational methods of therapy. For example, in the USSR a quantitative topochemical analysis of retinal nucleic acids has prompted the development of an effective methods for treating tapetoretinal dystrophy - a grave disease leading to blindness in childhood or adolescence.

In the last few years there has developed in microscopic anatomy a three-dimensional analysis of organ structure. It should be noted that an organ is not only part of the entire organism, but is itself an entity. 
502

Zhdanov

Although its name might give reason to regard it as an exclusively analytical science, presentday anatomy employs both analytical and synthetical methods in their unity. While breaking down an organism into its constituent apparatuses, systems, organs, tissues and cells, anatomy remembers that in the living organisms these constituents do not coexist separately but continuously interact and influence one another morphogenetically.

The construction of a given organ represents a morphophysio-logical and morphochemical unity of the structural elements of the organ, such as osteons and chondrones, muscle tissue bundles, segments of the liver and of other glands, lung lobules, nephrons, and other elements, whose functions make up the function of a given organ as a microanatomic polymeric single whole. The integration of individual structural-functional elements into a whole organ structure is largely dependent on connective tissue stroma. This is peculiar to each organ. True, this peculiarity has not so far been adequately studied, despite the great role played by organic connective tissue in pathological processes, especially in the interplay of the "local and the "general " during the pathogenesis and the course of a disease. Within an organ, the stroma is intimately linked up with the intrinsic networks of blood and lymph vessels and the architecture of intrinsic innervation. The construction of an organ reflects its functions and determines many of the aspects of these functions in health and disease.

The localization and the progress of pathological processes are de-determined, among other factors, by the organ construction. It is impossible, e. g. to understand acinous tuberculosis of the lungs, degenerative events in the liver, the location and distribution of nephron lesions in nephritis, or the direction of bone fractures, unless proper consideration is given to the construction of the respective organs. The particular features of pathological processes occurring in the right and left hepatic segments are associated with the different architecture of the blood vessels and differing conditions of blood circulation therein. The location and distribution of tuberculous and carcinous lesions in a lung depend on the latter's architectonics and the functioning of its blood an lymph vessels.

A stereomorphological establishment of correct views on the structure of whole organs is one of the most important conditions for a comprehensive, synthetic understanding of interrelationships between organ structure and function in health and disease, proceeding

Anatomy and medicine

503

from the principle of the unity of the part and the whole. In this connection, stereology evokes considerable interest. With the aid of scanning and integrative devices it enables to give an exact quantitative characterization of organ construction (e. g. of the lungs) and to ascertain the ratio between the quantity and size of structural elements of a given organ. These elements are proportional to the number of vitally important functions performed by the organs and by the entire organism. The next step is to apply stereological methods to the study of pathological changes in organs.

Challenging problems of concern for biology and medicine are being tackled by neuromorphology. Electron microscopy, in conjunction with ultracytochemistry and parallel electrophysiological intracel-lular recording of electrical potentials has greatly contributed to our understanding of the functional morphology of nerve cells and synapses. Very exact data have been reported regarding the ultrastruc-ture and ultracytochemistry of subsynaptic membranes, both central and peripheral, that transmit excitatory and inhibitory impulses for synapses. Studies 
are in progress dealing with fine receptor structures. All this brings us ever nearer to the elucidation of the morphological substrates of memory and reasoning. Yet, despite my high appreciation of the possibilities of electron microscopy and ultrastructural neurochemistry. I as an anatomist would like to emphasize that the integrative function of the central nervous system is determined by its complex organization on the supracellular level and, for that reason, an adequate understanding of this organization cannot be attained through the use of electron microscopy and cytoneurochem-istry alone. Interrelationships of nerve cells can be revealed only by combining electron microscopy with the use of classical silver impregnation procedures. A number of interesting studies have been undertaken in this direction, resulting in the discovery of unusual axo-axonal, dendro-dentritic, somato-dendritic and other junctions, which makes it necessary to review the concepts on interneuronal functional relations and to try and construct various neurocybernetic models of the brain and of its individual morphophysiological systems. The complex organizational pattern of the nervous system and of its integrative function will be better understood when the details of nervous system development under the effect of hereditary and environmental factors, training, and exercise will be known. Indeed, the mechanisms whereby the genetic determinism of numerous and

504

Zhdanov

very accurate connections within the central nervous system are realized, still remain quite mysterious, all the more so because these mechanisms develop, in part at least, before the abovementioned connections become functional.

In brain research, a number of investigations using labelled atoms and other markers have been carried out to trace the movement of neural elements in the developing brain. This is a highly important investigative path also for neuropathology, enabling more precise information to be obtained on central nervous system organization at the supracellular level.

The development of intravital transillumination and television microscopy and cinemicrography has given a fresh impetus to the study of blood and lymph microcirculation. The permeability of vessel walls is studied by observing the passage of free dyes and dyes bound to protein molecules through the endothelium. These investigations are now linked up with the elucidation of the obscure aspects of the general pathology of inflammation.

The macroscopical and macro-microscopical anatomic and topographic investigations of the individual and age- associated variability of the form, structure and topography of human organs, which are indispensible for improving the diagnostic and surgical methods, are now conducted with using present-day mathematical methods for variance analysis, as well as computers. X-ray anatomy is beginning to transform on the basis of electron-optical amplifiers of X-ray images of soft tissues and on the basis of colour roentgenography. The new techniques for studying movement have given rise to anatomic investigations aimed at creating a new discipline bioengineer-ing of the human body on earth and in outer space. Studies are under way to test the resistance of the skeleton and soft tissues to stresses and vibrations under ordinary and extreme conditions of gravitation and acceleration.

In embryology, the problem of greatest current interest is that of determination. It received a great impetus when the role of nucleic acids in protein synthesis and the role of enzymes and histones in the control of nucleic acids themselves had been ascertained. The problem of cell and tissue specialization has become one of the cytoplasmic control of the genetic apparatus of the cell nucleus. Experiments with transplantation of tissue cell nuclei to enucleated ova are well known. 


\section{Anatomy and medicine}

\section{5}

The concepts of the integrity of a developing organism and of the integrating (endocrine, neural, immunological) factors of embryo-genesis are becoming less vague and more complete. These factors now appear to control the genes in the course of development.

The theory of critical periods of development is of immediate interest for medicine. According to this theory, e. g. the developmental anomalies observed in rediation embryology depend on the particular developmental stage, the embryo was passing through at the moment of irradiation. This theory lays the foundations for elaborating recommendations regarding antenatal protection of the foetus and drug tests on embryos, which would permit specification of contraindications to the use of drugs by pregnant women.

Medicine has the right to expect from embryology a more concrete deciphering of factors and agents that determine tissue and organ development during embryogenesis. When known, these factors and agents may be used with advantage in the prevention of developmental anomalies and foetal diseases. This refers to genetic factors, to various forms of cell-tissue interactions and, finally, to environmental influences which in mammals and humans are mediated through the maternal organism. Embryology and pathology have a broad field of contact and overlap. Study of the states of, and of the morphogenetic processes in the embryo at the borderline between health and disease, as well as study of the reversibility of such processes, are of direct concern for prenatal prophylaxis. 